\title{
संगीत वादन में ताल एवं लय का महत्व
}

डॉ. कुलदीप कुमार

सह आचार्य, संगीत वादन, खालसा कालेज, पटियाला

\section{ABSTRACT}

The combination of singing, playing and dancing is known as 'music'. In India dance is declared outside the term Music due to some technical reasons. Singing and playing has been considered only in the music. Singing and playing are complementary to each other. Therefore, without one of the two, the existence of another is almost non-existent. Tala and Rhythm are important in the field of Music. An attempt has been made to tell about the rhythm and rhythm related to music playing.

Key words : Rhythm, Laya, Instrumental Music

आज का युग विज्ञान एवं तकनीक का युग है। विज्ञान एवं तकनीक ने प्रत्येक क्षेत्र में मनुष्य को एक नयी दिशा प्रदान की है। इस आधुनिक बढ़ रही वैज्ञानिक एवं तकनीकी प्रगति के कारण समूचे विश्व ने एक गाँव-का-सा रूप धारण कर लिया है। इस विश्व-रूपी गाँव में प्रत्येक देश की अपनी अलग संस्कृति है। इस संस्कृति के विकास में संगीत (गायन, वादन एवं नृत्य) ने अपनी महत्वपूर्ण भूमिका अभिनीत की है। भारत की यदि बात करें तो यहाँ सिन्धु घाटी की सभ्यता के समय से ही गायन, वादन एवं नृत्य तीनों कलाओं में ही संगीत का अत्योत्तम विकास हुआ है।

संगीत मानवीय लय एवं तालबद्ध अभिव्यक्ति है। भारतीय संगीत अपनी मधुरता, लयबदधता तथा विविधता के लिए जाना जाता है। वर्तमान भरतीय संगीत का जो रूप दष्टिगत होता है, वह आधुनिक युग की प्रस्तुति नहीं है, बल्कि यह भारतीय इतिहास के प्रारंभ के साथ ही जुड़ा हुआ है। वैदिक काल में ही भारतीय संगीत के बीज पड़ चुके थे। सामवेद उन वैदिक ऋचाओं का संग्रह मात्र है जो गेय है। प्राचीन काल से ही ईश्वर-आराधना हेतु भजनों के प्रयोग की परम्परा रही है। यहाँ तक कि यज्ञादि के अवसर पर भी समूहगान होते थे। ध्यान देने की बात है कि प्राचीन काल की अन्य कलाओं के समान ही भारतीय कला भी धर्म से प्रभावित थी। वास्तव में भारतीय संगीत की उत्पत्ति धार्मिक प्रेरणा से ही हुई है, परन्तु धीरे-धीरे यह धर्म को तोड़ कर लौकिक जीवन से सम्बन्धित होती गयी और इसी के साथ नृत्य कला, वाद्य तथा गीतों के नये-नये रूपों का आविष्कार होता गया। कालांतर में नाट्य भी संगीत का एक हिस्सा बन गया। समय के साथ संगीत की विभिन्न धराएं विकसित होती गयीं, नये-नये राग, वाद्य यन्त्र और कलाकार उत्पन्न होते गये। भारतीय संगीत जगत अनेक महान विभूतियों के योगदानों के परिणामस्वरूप ही इतना विशाल रूप धारण कर सका है।

उपरोक्त चर्चा के आधार पर स्पष्ट है कि गायन, वादन एवं नृत्य इन तीनों कलाओं में से वादन का भी उतना ही महत्व-पूर्ण स्थान है जितना कि गायन तथा नृत्य का। यही कारण है कि आधुनिक काल में प्राचीन काल से प्रचलित धारणा कि गायन, वादन एवं नृत्य में से गायन को सर्वोत्तम स्वीकृत किया गया है तथा गायन के अधीन वादन, वादन के अधीन नृत्य को माना गया है, गलत हो गयी है, अपितु आज-कल गायन एवं वादन को एक-दूसरे के पूरक स्वीकृत किया गया है। 
गायन के बिना वादन तथा वादन के बिना गायन अधूरा-सा प्रतीत होता है। इस संगीत वादन में जितना महत्व स्वर का है, उतना ही महत्व ताल एवं लय का भी है।

संगीत वादन में ताल एवं लय' का महत्व विषय को स्पष्ट करने के लिए निम्नोक्त शीर्षकों-उपशीर्षकों के अंतर्गत चर्चा की जा रही है-

- संगीत

- संगीत वादन

- ताल

- लय

- संगीत वादन में ताल एवं लय का महत्व।

संगीत

"गीतम् वाद्यम् तथा नृत्यं त्रयम् संगीतम उच्यते"

गायन, वादन एवं नृत्य ये तीनों कलाओं के मिश्रण को संगीत कहा जाता है। वास्तव में ये तीनों कलाएं एक-दूसरे से स्वतन्त्र हैं, परन्तु स्वतन्त्र होते हुए भी गायन के अधीन वादन और वादन के अधीन नृत्य है। प्राचीन काल में ये तीनों कलाओं का प्रयोग एक साथ किया जाता थां 'संगीत' शब्द दो शब्दों के सुमेल से बना है। 'सं+'गीत' 'स' से अभिप्राय सम्यक् अथवा भली-भाँति तथ 'गीत' का भाव गीत अथवा गाना या यों कहें, किसी गीत को उत्तम ढंग से गाने को 'संगीत' कहते हैं। इन तीनों कलाओं में गायन को सर्वोच्च माना गया है। ये तीनों कलाएं निम्नलिखित हैं-

(1) गायन-किसी गीत द्वारा या गा कर अपने मन के भावों को व्यक्त करने की कला को 'गायन' कहते हैं।

(2) वादन-किसी वाद्य को बजाते हुए अपने मनोभावों को अभिव्यक्त करने की कला को वादन कहते हैं।

(3) नृत्य-शारीरिक हाव-भावों द्वारा अपने मन के भावों को अभिव्यक्त करने की कला को नृत्य कहते हैं।

संगीत में जहाँ एक ओर स्वर की प्रधानता रहती है, वहीं दूसरी ओर ताल व लय के बिना संगीत की कल्पना तक नहीं की जा सकती। डा. शोभा कुदेशिया के विचारानुसार, "भारतीय संगीत के आदयाचार्य भरत मुनि ने गांधर्व अर्थात् संगीत को 'स्वरतालपदात्मकम्" कहा है। संगीत में स्वर, पद और ताल तीन तत्वों की आवश्यकता होती है। स्वर का तात्पर्य संगीतोपियोगी ध्वनि से है, पद का अर्थ शब्द सम्पत्ति से है तथा काल का अर्थ काल मापक साधन से है। संगीत में स्वर, शब्द और ताल का अपूर्व मिलन ही संगीत की उत्कृष्टता का द्यौतक है। ताल मूलतः लय को प्रकट करने की क्रिया है। ${ }^{1}$

ताल एवं लय का प्रत्यक्ष सम्बन्ध संगीत वादन से हैं अतः ऊपर की गयी चर्चा से एक बात तो स्पष्ट हो ही गयी है कि गायन, वादन एवं नृत्य इन तीनों कलाओं में गायन सर्वोत्म नहीं, अपितु गायन एवं वादन एक-दूसरे के पूरक हैं, क्योंकि ताल एवं लय के अतिरिक्त अन्य भी कई चीज़े हैं जो गायन ने वादन से ग्रहण की हैं जिनके बारे में चर्चा आगे की जा रही है। 
संगीत वादन

संगीत वादन से तात्पर्य है, बजाना। जब हम किसी वाद्य पर कुछ भी बजाते हैं, उसे संगीत वादन कहा जाता है। संगीत वादन दो प्रकार का होता है-स्वर वादन एवं ताल वादन। सवर वादन में सितार, सरोद, वीणा सारंग तथा ताल वादन में तबला, पखवज आदि वाद्य आते हैं।

उ. शाहिद परवेज़ के अनुसार, "हमारे घराने में सिर्फ साज़ छेडेंगे तो पता लग जाएगा कि ये इस घराने का है। पहले सितार में दाएं हाथ का काम ज्यादा हुआ करता था, पर धीरे-धीरे जैसे एक मिसाल कहते थे कि गाना उत्तम, बजाना मध्यम। ये मिसाल की वजह ही ये थी कि संगीत को स्थापित करने के लिए इसको जिसका जितना ज्यादा है, उस हिसाब से यह मिसाल बनाई गयी। यह मिसाल आज के लिए नहीं। यह उस ज़माने की मिसाल थी। मेरे बुजुर्गों ने उसको ध्यान में रख कर साज़ को जो उसकी कमियाँ थीं, जिसकी वजह से यह गले से पीछे रहता था, उसको बहुत हद तक कम किया। ${ }^{\prime 2}$

ऊपर की गयी चर्चा से स्पष्ट है कि साज़ों पर न केवल तंत्रकारी अंग से ही काम हो रहा है, अपितु गले की समस्त बारीकियो को भी सितार जैसे वाद्य पर आज के युग में बखूबी प्रदर्शित किया जा रहा है। संगीत वादन में ताल एवं लय का भी बहुत महत्व है जिसके बारे में आगे विचार किया जा रहा है।

ताल

संगीतार्णव' संस्कृत ग्रंथ में तांडव (पुरुष) नृत्य से ता' तथा लास्य (स्त्री) नृत्य से 'ला' वर्णों के संयोग से 'ताल' शब्द की व्योत्पत्ति दर्शाई गयी है। 'संगीत दर्पण' में 'ताकार' से शंकर या शिव और 'लकार' से पार्वती या शक्ति, दोनों का योग 'ताल' कहा गया है। संगीत में काल की नियमित गति या लय ताल को जन्म देती है। समय के साधन को ताल कहा जाता है। संगीत में गायन, वादन एवं नृत्य की क्रियाओं में जो समय लगता है, उसके मापने कोक ताल कहा जाता है। दूसरे शब्दों में ताल, काल को मापने का पैमाना है। भिन्न-भिन्न मात्राओं कमे समूह से भिन्न-भिन्न तालों की रचना होती है। ताल संगीत को संचालित करता है। ताल का निर्धारण होने से गायक, वादक एक मर्यादा में बाँधे जाते हैं। ग्रंथों में ताल के बिना गायन, वादन एवं नृत्य की तुलना मस्त हाथी से की गयी है तथा ताल को अंकुश की उपमा दी गयी है। ताल संगीत को अनुशासित करते उसके संगठित रूप, स्थाईपन एवं दस्तकारिता से श्रोताओं को आनन्दविभोर कर देता है। डा. रचना भृगुवशी के अनुसार-

$$
\text { "पूर्णताल प्रवृत्तिस्यात् पश्चात् गीतादिरुपते । }
$$

ताल संगीत का पोषक तत्व है, इसी कारण ताल को विष्णु कहा गया है। श्रीमति सुसान लैंगर के अनुसार, "The essence of rhythem is tension and resolution" अर्थात अपेक्षित घात की उत्सुकता तथा आघात आने पर उस उत्सुकता एवं तनाव का विसर्जन यही लय का सार है। 'सम' लय का केन्द्र स्थल होता है। संगीत का वो आनन्ददायक तत्व मानो इसी 'सम' में छिपा रहता है, जो कुशल गायक, वादक एवं नर्तक की प्रत्यक्ष क्रिया का आधार लेकर सहस्त्रों धाराओं में प्रस्फूटित होता है। अतः इसी के द्वारा भारतीय संगीत अन्य संगीत परम्पराओं में श्रेष्ठ स्थान रखता है। आधुनिक भारतीय संगीत में तालों की प्रतिष्ठा मुख्य रूप से तबला, पखवज, मृदंग, घुंघरू आदि घन वाद्यों पर की जाती है ${ }^{4}$ 
लय

समान चाल, गति, स्पीड को संगीत की भाषा में लय कहा गया है। ईश्वर की रची हुई कायनात एक लय में चल रही है। सूर्य, चन्द्रमा, वायु, जल, पृथ्वी आदि शक्तियाँ एक गति में क्रियामान हैं। दिन-रात पृथ्वी की गति पर आधारित हैं। मानवीय शरीर भी गतिशील है। इसके अंग भी एक विशेष लय में कार्य कर रहे हैं। समय की गति को मापने के लिए घड़ी का आश्य लिया जाता है। घड़ी की टिकटिक एक स्पीड में होती है। इस टिकटिक को हम लय कहते हैं।

"श्रुति अधीन गायन बन्यौ श्रुतिन बसे रस जान लय सो प्रकटाये पुरा, रूपक चतुर सुजान'

$$
\text { पं. भातखंडे }
$$

'लय' शब्द की उत्पत्ति ली धातु से हुई है जिसका अर्थ है एक हो जाना। अमर-कोष में लिखा है, "लय साम्यम्।" अर्थात् जब दो के बीच में एकरूपता या साम्यय इस प्रकार सम्पन्न हो जाए कि उसका अंतराल न कम हो और न अधिक तो उसे लय कहते हैं। ${ }^{5}$

ताल का सम्बन्ध लय से है तथा लय संगीत का आधार है। समय के किसी भी विभाग की एक समान गति को लय कहा जाता है। इसी प्रकार संगीत में भी गायन, वादन एवं नृत्य का सम्बन्ध लय से है। संगीत में लय का महत्व बताते हुए कहा गया है कि श्रुति माता है तो लय पिता है। लय संगीत का वह साधान है जिससे आनन्द तथा रस का स्रोत दूसरों तक पहुचता है। गायन, वादन एवं नृत्य की क्रियाओं पर जो समय लगता है, उसकी चाल या गति को लय कहा जाता है। लय एक समानन्तर चाल होती है जिसमे तीन प्रकार हैं-

(क) विलंबित लय- बहुत धीमी चाल अथवा गति को विलंबित लय कहा जाता है। इसे ठा लय भी कहते हैं। गायकी में बड़े ख्याल, ध्रुवपद एवं धमार आदि इसी लय में गाए जाते हैं। विलंबित लय के कुछ ताल हैं-झूमरा, आड़ाचार ताल, तिलवाड़ा, एकताल, चारताल आदि।

(ख) मध्य लय- साधारण लय जो न अधिक धीमी तथा न ही तेज हो, मध्य लय कहलाती है। मध्य लय की तुलना हम घड़ी के हर सैकिण्ड की टिकटिक की ताल से कर सकते हैं। गायकी में छोटा ख्याल, गीत तथा भजन आदि इसी लय में ही गाए जाते हैं। वादन में द्रुत अथवा रज़ाखनी गतें, धुनें आदि इस लय में बजाई जाती हैं। इस लय की तालें हैं-तीनताल, झपताल, कहरवा, दादरा आदि।

(ग) द्रुत लय- मध्य लय से दुगुनी तेज़ लय को द्रुत लय कहा जाता है। गायकी में तराने, ख्याल आदि इसी लय में गाए जाते हैं। द्रुत लय की तानों को तेज करके ही गाया-बजाया जाता है। उदाहरणार्थ एक मिनट में एक-सी चाल से यदिसाठ तक गणना की जाये तो वह मध्यलय बन जाती है। इसी तरह एक मिनट में यदि तीस तक गणना करें तो विलंबित लय तथ यदि 120 तक गणना करें तो वह द्रुत लय बन जाएगी।

संगीत वादन में ताल एवं लय का महत्व

संगीत में ताल एवं लय का अत्याधिक निकटवर्ती सम्बन्ध है। जब हम वादन की बात करते हैं तो उसमें लय का सदा महत्व रहता है। या यों कहें कि जब कोई वादन वादक द्वारा किया जाता है, उस समय हो रहे मंच-प्रदर्शन द्वारा यह देखा जाता है कि जो भी प्रस्तुतिकरण कलाकार द्वारा किया जा रहा है, उसमें तकनीकी पक्ष से विभिन्न लयकारियों का ताल से क्या सम्बन्ध है। यह भी 
ध्यान रखा जाता है कि हो रहे ताल वादन में सम्बन्धित ताल तबले का है अथवा पखावज का, क्योंकि जो ताल पखावज से सम्बन्धित होते हैं, उनमें गंभीर लयकारियों का प्रदर्शन किया जाता है जबकि जो ताल तबले से सम्बन्धित हैं, उनमें समयानुसार स्वतन्त्र वादन दौरान पेशकार, कायदा, टुकड़ा, रेला विभिन्न लयों में तिहाइयाँ तथा इनके अतिरिक्त सुंदर परनों का बहुत कुशलतापूर्वक प्रयोग किया जाता है। इस विचारधारा अनुसार जो हिंदुस्तानी संगीत के 40 सिद्धाँत हैं, उनमें यह देखा जाता है कि ताल के बिना लय तथा लय के बिना ताल संभव नहीं है। एक अहम पक्ष यह भी सामने आता है कि जब स्वतन्त्र वादन किया जाता है तो ताल के सही गलत स्थान के लिए नगमे का प्रयोग किया जाता है तो इस सिद्धांत को सिद्ध करता है कि जिस शैली का वादन लयकारी के रूप में किया जा रहा है, उसकी मात्राएं लय में पूरी आ रही है या नहीं। इसके अतिरिक्त एक अन्य पक्ष भी सामने आता है कि जब गायन कला दौरान गायन का प्रदर्शन किया जाता है तो उस समय भी जब गुणीजन गायन की लय रखते हैं तो तबले वाले गुणीजन भी उसके अनुसार की लय रखते हैं। भाव चाहे वह गायन हो अथवा वादन, दोनों में लय की सांझ है। जब ताल का प्रारंभ करना हो तो लय दिखाने के लिए ताल के विभागों की ओर ही ध्यान दिया जाता है। कहने का तात्पर्य यह है कि विभागों के अनुसार उसमें लयकारी का प्रयोग करते ताल के चलन का भी मुख्य रूप से ध्यान दिया जाता है। हिंदुस्तानी शास्त्रीय संगीत की ताल वादन परम्परा में गिनती की ओर विशेष ध्यान दिया जाता है। इस गिनती को ध्यान में रखते हुए यह भी ध्यान दिया जाता है कि हम दोगुन अथवा चौगुन किस लय में प्रदर्शन करना है, उसके साथ-साथ कितनी मात्राओं की तिहाई का वादन करना है, किस ताल का प्रयोग करना है, तकनीकी पक्ष से इस तरह नहीं किया जा सकता कि किस मात्रा से उस तिहाई को उठाना है तथा किस तरह इच्छा से बजा देना है।

दरअसल यदि किसी ताल में किसी शैली का वादन किया जाता है तो यह भी ध्यान रखा जाता है कि यदि हम चक्रदार परन बजानी है या फिर फरमाइशी चक्रदार अथवा कमाली चक्रदार परन का प्रयोग करना है तो उस ताल में ही सही होगी जिस ताल के लिए उसे बनाया गया है और यहाँ इस बात का ध्यान देना चाहिए कि यदि गिनती के आधार पर कमाली चक्रदार तीनताल के लिए बनी है तो उसे किसी अन्य ताल में जैसे झपताल, रूपक ताल में गिनती के आधार पर तो वादन किया जा सकता है, परन्तु तकनीकी पक्ष से उसे फरमाइशी चक्रदार परन नहीं कहा जा सकता, क्योंकि लयकारी तथा गिनती के हिसाब से वह परन तीनताल के लिए ही बनी है न कि किसी अन्य ताल के लिए। जब हम लयकारी की बात करते हैं तो इस सिदधांत का भी मुख्यतः ध्यान रखा जाता है कि जैसे तालवादन में दोपल्ली तिहाई को दो तरह की लय में वादन किया जाता है, उसमें तिपल्ली तिहाई में तीन प्रकार की लय, चौपल्ली तिहाई में चार प्रकार की लय तथ पंचपल्ली तिहाई में पाँच प्रकार की लय। इसके बिना सप्तर्षि तथ दर्जे की गत में भिन्न-भिन्न लयों का प्रयोग गुणीजन आम ही करते हैं। एक अन्य पक्ष यह भी देखा जाता है कि जब कायदे का वादन किया जाता है, उसमें दोगुन, चौगुन का प्रयोग गुणीजन आम ही करते हैं। इसी तरह तंती साज़-वादन की परम्परा में भी लय का मुख्य महत्व है तथा बंदिश की लय को बढ़ा कर ही अंत में जब अति द्रुत लय हो जाती है तो झाले का वादन भी एक विशेष लय में किया जाता है। वैसे तीन प्रकार की लय होती है-विलंबित, मध्य तथा द्रुत। इसके अतिरिक्त दो प्रकार की लय और भी स्वीकृत की जाती है जो कि अति विलंबित तथा अति द्रुत भी हो सकती है। इस तरह चाहे वह 
तंती साज़ वादन हो, चाहे तबला वादन हो, चाहे पखावज वादन, उसमें लयकारियों के रूप में आड़, क्वाड़ आदि लयों का प्रयोग भी बहुत सुंदर लय में किया जाता है।

\section{निष्कर्ष}

अंत में हम निष्कर्षतया कह सकते हैं कि ताल एवं लय का संगीत वादन से बहुत गहरा सम्बन्ध है। ताल तथा लय सिक्के के ऐसे दो पहलू हैं जिनमे बिना एक-दूसरे का अस्तित्व संभव नहीं है। अतः संगीत वादन में इन दोनों का ही अति महत्वपूर्ण स्थान है।

\section{संदर्भ}

प्राचीन ताल के परिपेक्ष्य में वर्तमान तबलावादन', कुदेशिया, शोभा डा., राधा पब्लिकेशन, नई दिल्ली, प्रथम संस्करण-2012, प्रथमाध्याय-'ताल का क्रमिक विकास एवं महत्व' पृष्ठ-1

दूरदर्शन के डी.डी. राज्यसभा से प्रसारित कार्यक्रम 'शख्सीयत' में उ. शाहिदपरवेज़ से उद्घोषिका समीना की भेंटवार्ता के फलस्वरूप प्राप्त जानकारी के आधार पर।

'भारतीय तालों का शास्त्रीय विवेचन', सेन, अरुण कुमार 'उा', मध्य प्रदेश हिंदी ग्रंथ अकादमी, भोपाल, प्रथम संस्करण-1973, पृष्ठ-47

'हिन्दुस्तानी शास्त्रीय संगीत में तन्त्र-वादन शैलियाँ, भृगुवशी, रचना 'डा.', कनिष्क पब्लिशर्स, डिस्ट्रीब्यूटर्स नई दिल्ली, प्रथम संस्करण-2014,

'संगीत सार', मानकरण, वीणा, राज पब्लिशर्स (रजि.), अड्डा टांडा, जालंधर शहर, लय और ताल, पृष्ठ-33 